\title{
Efecto del volumen de suelo húmedo en riego localizado para el cultivo del avellano (Corylus avellana L.)
}

\author{
J.R. Gispert' ${ }^{1}$, F. Ramírez de Cartagena ${ }^{2, *}{ }^{,}$J.M $^{a}$ Villar $^{3}$ y M. Rovira ${ }^{1}$ \\ 1 Institut de Recerca i Tecnologia Agroalimentàries (IRTA). Mas de Bover. Ctra Reus - El Morell, Km 3,8 \\ 43120 Constantí (Tarragona). España \\ 2 Departament d'Enginyeria Quimica, Agrària i Tecnologia Agroalimentària. Universitat de Girona. \\ Campus Montilivi, 17071 Girona. España \\ 3 Departament de Medi Ambient i Ciències del Sòl. Universitat de Lleida. Av. Alcalde Rovira Roure, 191, \\ 25198 Lleida. España
}

\section{Resumen}

Se determinó el comportamiento productivo, vegetativo y la calidad del fruto en avellano (Corylus aveIlana L.), como respuesta a 4 volúmenes de suelo húmedo que recibieron la misma cantidad de agua de riego, 3 de ellos (VSH-6, VSH-15, VSH-33\%) generados mediante riego por goteo y 1 (VSH-70\%) con difusores, expresados en términos de volumen potencial de exploración radicular. El experimento se realizó sobre el cultivar 'Pauetet', en una finca comercial en Tarragona (España). El volumen de suelo húmedo más reducido (6\%) generó la producción menor de avellana en cáscara. Asimismo el mayor porcentaje de volumen de suelo húmedo $(70 \%)$, generado mediante riego por microdifusión, dio lugar a manifestaciones de estrés hídrico debido a los bajos contenidos de agua del suelo y probablemente mayor evaporación, siendo las producciones en cáscara similares a las alcanzadas con el volumen de suelo húmedo del $6 \%$. La mayor producción de avellana en cáscara y el mayor crecimiento vegetativo de los brotes se alcanzaron con un porcentaje de suelo húmedo del $33 \%$. De acuerdo con los resultados obtenidos en los parámetros productivos y de calidad sería recomendable el riego por goteo para obtener un porcentaje mínimo de volumen de suelo húmedo del $33 \%$.

Palabras clave: productividad del agua, gestión del riego, riego por goteo, riego por difusión.

\section{Abstract \\ Effect of wet soil volume in microirrigation for hazelnut production (Corylus avellana L.)}

The productive and vegetative behaviour and fruit quality of hazelnut (Corylus avellana L.) was determined in response to 4 wet soil volumes receiving the same amount of water irrigation, 3 of them (6, $15,33 \%)$ generated by drip irrigation and $1(70 \%)$ with microsprayers, expressed in terms of potential root exploration volume. The experiment was conducted on the cultivar 'Pauetet' in a commercial farm in Tarragona (Spain). The smaller volume of wet soil (6\%) generated the lower production of hazelnut in shell. Also the highest percentage of wet soil volume (70\%) generated by microsprayer irrigation showed water stress due to the low water content of the soil and, probably, a higher evaporation of the soil surface, with hazelnut in shell productions like those achieved with the volume of $6 \%$ wet soil. In-

* Autor para correspondencia: francisco.ramirez@udg.edu http://dx.doi.org/10.12706/itea.2015.008 
creased production of the hazelnut in shell and the greater vegetative shoot growth were achieved with a percentage of $33 \%$ wet soil volume. According to the results obtained in the production and quality parameters it would be advisable the drip irrigation for a minimum percentage of $33 \%$ wet soil volume.

Key words: water productivity, irrigation management, drip irrigation, micro-sprayer irrigation.

\section{Introducción}

La producción de avellana en cáscara a nivel mundial alcanzó, en 2012, las 914.447 t (FAOSTAT, 2014a), con Turquía como principal país productor (660.000 t). La Unión Europea (UE), con 118.172 t representa el $13 \%$ de la producción mundial, donde Italia y España son los principales países productores ( $84 \%$ de la UE). Durante muchos años, España fue el cuarto país productor mundial de avellanas, después de Turquía, Italia y EE. UU. (Tous et al., 2001). No obstante, actualmente ocupa el octavo lugar con 13.900 t en 2012 (FAOSTAT, 2014a), debido a la importante presencia de otros países: Azerbaiyán, Georgia, Irán y China. La producción mundial de avellana en cáscara alcanzó en 2011 un valor de 2.242 millones de USD (Dólar americano), en Europa 476 millones de USD y en España de $41 \mathrm{mi}-$ llones de USD (FAOSTAT, 2014b).

En España, la superficie dedicada a este cultivo en 2013 era de 14.777 ha de las cuales el 90\% estaban en Cataluña (Ministerio de Agricultura, Alimentación y Medio Ambiente, 2013). En los países mediterráneos, las variedades que se cultivan son las tradicionales y son las mismas desde hace tiempo. La innovación está relacionada con el manejo de nuevas técnicas de cultivo (Fideghelli y De Salvador, 2009). A nivel mundial las áreas geográficas con mayor producción de avellana, corresponden a regiones de abundante pluviometría, a menudo superior a $1000 \mathrm{~mm} / a n ̃ o$ (Giresun, Trebisonda en Turquía; Nápoles, Viterbo y Alba en Italia, Oregón en EE. UU.). La provincia de Tarragona en España es una excepción, con cerca de $500 \mathrm{~mm}$ de lluvia/año (Germain y Sarraquigne, 2004). El avellano requiere una pluviometría anual cercana a los $800 \mathrm{~mm}$, bien distribuidos, para poder producir buenos resultados productivos en ausencia de riego (Romisondo et al., 1988, Tous y Rovira, 2004, Rovira y Aletà, 2012). Otros autores indican necesidades superiores a 1000 mm (Silva et al., 2005, Grau, 2009).

La escasez de agua para el riego, endémica en el área de cultivo de Cataluña (Noreste de España) y la mayoría de las zonas mediterráneas, no ha propiciado una adecuada cultura para dar respuesta a las necesidades hídricas de ese cultivo. Sin embargo, a mediados de los años 60 , cuando irrumpen en la agricultura los sistemas de microirrigación, empieza a considerarse un uso más eficiente del agua aplicada. Actualmente, el $67 \%$ de las plantaciones de avellano en Cataluña se encuentra en régimen de regadío (Baiges et al., 2012).

Girona et al. (1994), indican la alta sensibilidad de esta especie a las reducciones del riego durante el verano, siendo la falta de agua en este período el motivo del importante descenso en la producción de grano por hectárea al reducirse el tamaño de la cáscara. El avellano es una especie con un comportamiento anisohídrico, tal como se deduce de los trabajos de Girona et al. (1994) y Marsal et al. (1997). Por este motivo, Gispert et al. (2005), indican la necesidad de regar desde abril a septiembre para obtener una buena respuesta productiva y de calidad del grano, además, si el riego se aplica sólo en verano o si se reduce en más del $50 \%$ la dotación de riego en esta estación, la calidad del grano se ve afectada.

En el diseño de los sistemas de riego localizado el establecimiento del volumen de suelo húmedo (VSH) adecuado es crítico para 
conseguir un rendimiento del cultivo y una eficiencia de aplicación del agua óptimos. En este sentido, Cohen et al. (1992) presentan como factor negativo para la buena productividad del avellano el escaso número de emisores ( 1 a 2 por árbol) en más de la mitad de explotaciones de Tarragona (España) y la escasa superficie de suelo mojado que ello representa. Gispert et al. (1996), para una aplicación anual de riego de $4.000 \mathrm{~m}^{3} / \mathrm{ha}$, aconsejan una superficie húmeda del suelo de alrededor del $40-60 \%$ del área sombreada por el avellano, a una profundidad de 30-40 $\mathrm{cm}$, como adecuada para conseguir una buena respuesta vegetativa y productiva. Asimismo, De Salvador y Monastra (1997) indican la necesidad de humedecer un amplio volumen de suelo, mediante microaspersión, para obtener una adecuada respuesta fisiológica y productiva del avellano.

La forma y dimensiones del VSH dependen del caudal del irrigador, del volumen de emisión, de las características del suelo y del contenido inicial del agua. Bennett (2002) indica la gran diferencia de formas que se producen en el volumen de suelo húmedo al variar la frecuencia de aportación del agua de riego en almendros, observando que aumenta el tamaño del VSH al incrementar la frecuencia del riego por el movimiento lateral del frente húmedo en los períodos de descanso entre pulsos consecutivos. Bresler et al. (1971) y Bresler (1978) señalan que el caudal del emisor influye en la forma del volumen de suelo húmedo, aumentando el radio y disminuyendo la profundidad a medida que aumenta el caudal de emisión. En las ecuaciones simplificadas de Schwartzmass y Zur (1986) para calcular el radio y la profundidad mojada, también la profundidad disminuye y el radio aumenta a medida que aumenta el caudal de emisión y disminuye la conductividad hidráulica saturada. Keller y Bliesner (1990), Angelakis et al. (1993) en dos suelos de distinta textura, Gispert y García
(1994) en un suelo de textura franca y Gispert (2008), observan el mismo comportamiento del caudal de emisión. Sin embargo, en los resultados obtenidos por Arbat et al. (2003) en un suelo Typic Calcixerepts (Soil Service Staff, 2010) utilizando goteros de 2, 4, 8,5 y $25 \mathrm{~L} \mathrm{~h}^{-1}$, el caudal del emisor tuvo menos importancia sobre el radio y profundidad mojada que la presencia de distintos horizontes edáficos o que el contenido de agua inicial del suelo. El código Drip-Irriwater (Arbat et al. 2013) permite determinar de manera simple y rápida las dimensiones del volumen de suelo húmedo y sus contenidos de agua en condiciones de riego por goteo.

Se concluye que el avellano es sensible a la falta de agua en determinados períodos fisiológicos de su cultivo y a un número reducido de emisores. También se constata que los estudios realizados durante la última década (Bignami et al., 2009 y 2011) en relación con el riego del avellano que permitan mejorar la eficiencia en el uso del agua son muy escasos.

El objetivo planteado en el presente trabajo fue determinar la respuesta productiva, vegetativa y de calidad del fruto del avellano para distintos porcentajes de volumen de suelo húmedo.

\section{Materiales y métodos}

\section{Parcela experimental}

El estudio se realizó en una parcela tradicional de avellano en Alcover (Tarragona, España). La experiencia se realizó durante 4 años consecutivos (1992-1995) en una parcela de $3.766 \mathrm{~m}^{2}$ de avellano (Corylus avellana L.) de 21 años de edad con una densidad de plantación de 361 árboles ha-1 $(4,71$ x 5,88 m) de la variedad 'Pauetet' con polinizador 'Gironell', variedades que constituyen la base de la producción en Cataluña (Tous et al., 2001). 
Los avellanos, formados en mata tradicional con diversos pies, como es habitual en las plantaciones de Tarragona (Tous et al., 2001; Baiges et al., 2012), fueron podados de igual forma durante todo el período experimental y presentaban un volumen medio de copa de $56 \mathrm{~m}^{3}$. El porcentaje de ocupación respecto al marco de plantación era del $76 \%$.

El suelo se caracteriza por la presencia de gravas alóctonas a una profundidad media de 40-60 cm que limitan el crecimiento radicular y favorecen su desarrollo predominantemente horizontal, con un buen drenaje y permeabilidad. De acuerdo con el Soil Survey Staff (2010) el suelo se clasificó como Typic Xerorthents, con una profundidad efectiva de las raíces de unos $60 \mathrm{~cm}$, y características muy similares en todo el perfil respecto a $\mathrm{pH}$ $(7,8-7,9), \mathrm{CE}_{1: 5}\left(0,50-0,70 \mathrm{dS} / \mathrm{m}\right.$ a $\left.25^{\circ} \mathrm{C}\right)$, materia orgánica $(1,4-2,0 \%)$, textura USDA (franco-limosa), caliza activa (4-5\%) y contenido volumétrico de agua medio $(29,4 \%$ a $-20 \mathrm{kPa}$; $13,7 \%$ a $-1500 \mathrm{kPa}$ ).

Para el estudio se consideraron únicamente las campañas 1992, 1994 y 1995, puesto que en el verano de 1993 una fuerte tormenta ocasionó el arrastre por escorrentía de las avellanas ya caídas en el suelo, mezclando los frutos de los diferentes tratamientos imposibilitando el conteo.

El promedio anual de Iluvia y de evapotranspiración de referencia $\left(\mathrm{ET}_{0}\right)$, durante el período de experimentación (1992, 1994, 1995), fue de $488 \mathrm{~mm}$ y $1095 \mathrm{~mm}$, respectivamente. La media mensual de las temperaturas máximas para este período varió entre $3,2^{\circ} \mathrm{C}$ (enero) y $29,4^{\circ} \mathrm{C}$ (agosto).

\section{Programa de riego}

Las necesidades de agua de riego se calcularon semanalmente, considerando la evapotranspiración del cultivo (ET $)$ (Allen et al., 1998) a partir de la evapotranspiración de referencia $\left(\mathrm{ET}_{0}\right)$, según el método de Penman-Monteith, corregida por el coeficiente de cultivo $\left(\mathrm{K}_{\mathrm{c}}\right)$ y el coeficiente de reducción $\left(\mathrm{K}_{\mathrm{e}}\right)$, este último considera el porcentaje de ocupación del cultivo respecto al marco de plantación. La ET se obtuvo de la Estación Agrometeorológica del Centro Mas de Bover (IRTA), situada a unos 5 $\mathrm{km}$ de la parcela experimental.

Los coeficientes de cultivo $\left(K_{c}\right)$ empleados fueron aquellos considerados para el avellano en el centro Mas de Bover y que corresponden, mensualmente, a los siguientes valores entre marzo y septiembre [marzo $(0,30)$, abril $(0,40)$, mayo $(0,60)$, junio $(0,65)$, julio $(0,85)$, agosto $(0,75)$ y septiembre $(0,63)]$. El K e consideró igual a 1 al ocupar el cultivo una superficie superior al $70 \%$ del marco de plantación (Fereres, 1981). La lluvia efectiva fue establecida como el $80 \%$ de la lluvia total (Doorenbos y Pruitt, 1988). Las necesidades totales de riego fueron finalmente determinadas aplicando una eficiencia de riego de 0,90 de acuerdo a la uniformidad de riego obtenida en la misma parcela.

Con el fin de aplicar la misma dosis de agua en todos los tratamientos, se estableció el criterio de considerar un tiempo de riego distinto para cada tipo de emisor $(4,8,24$ y 50 $\mathrm{L} \mathrm{h}^{-1}$ ) y mantener la misma frecuencia de riego semanal en cada tratamiento. La frecuencia de riego se estableció según los requerimientos hídricos de la semana anterior.

\section{Tratamientos de riego}

Se establecieron 4 porcentajes de VSH, 6, 15, 33 y $70 \%$ del volumen total de suelo que podía ser explorado por las raíces del cultivo (volumen potencial de exploración radicular, VPER) mediante tres tipos de goteros autocompensantes $\left(4,8\right.$ y $\left.24 \mathrm{~L} \mathrm{~h}^{-1}\right)$ y un tipo de difusor $\left(50 \mathrm{~L} \mathrm{~h}^{-1}\right)$. Para cada tipo de gotero se instalaron 2 laterales de riego por fila de aveIlanos y 10 goteros /árbol (5 a cada lado), el difusor se estableció mediante un lateral de riego por fila y 1 difusor /árbol. Atendiendo 
los criterios de localización de los goteros respecto al tronco, indicados por diversos autores (Jiménez et al., 1977; Nuñez-Aguilar et al., 1980; Fernández et al., 1991), la experiencia se planteó manteniendo, para cada uno de los dos laterales de goteros en cada fila, un desplazamiento horizontal de 1,5 m del centro de cada avellano. El lateral para los difusores se situó a lo largo de la fila de avellanos y el difusor a 1,5 $\mathrm{m}$ del centro de cada árbol.

Variando el tiempo de riego en función del caudal suministrado por cada tipo de emisor se aplicó el mismo volumen de agua. Asi- mismo y en función del número de emisores por árbol se suministró una dosis de riego de 177 Lárbol en todos los casos (Tabla 1) variando su frecuencia semanal en función de la demanda evapotranspirativa de cada semana.

El diseño experimental fue planteado en 4 bloques al azar y 3 repeticiones para cada porcentaje de VSH. Cada una de las 16 parcelas elementales consistía de 3 árboles consecutivos controlados y un árbol guarda entre bloques. Un total de 68 avellanos estuvieron implicados en este experimento, 48 de los cuales fueron controlados.

Tabla 1. Volumen de agua de riego aportado para cada árbol (L) mediante los distintos emisores, radio mojado $(\mathrm{cm})$ y área mojada $\left(\mathrm{m}^{2}\right)$ a $30 \mathrm{~cm}$ de profundidad, para los distintos emisores y volúmenes aplicados al finalizar cada riego

Table 1. Volume of irrigation water given to each tree $(L)$ by the different emitters, wet radius $(\mathrm{cm})$, and wetted area $\left(\mathrm{m}^{2}\right)$ at $30 \mathrm{~cm}$ depth for different emitters and applied volumes after each irrigation

\begin{tabular}{|c|c|c|c|c|}
\hline \multirow{2}{*}{$\frac{\text { Emisor }}{\text { Caudal emisor }\left(\mathrm{L} \mathrm{h}^{-1}\right)}$} & \multicolumn{2}{|c|}{ Goteros } & \multicolumn{2}{|c|}{ Difusor } \\
\hline & 4 & 8 & 24 & 50 \\
\hline Tiempo (h) & 4,42 & 2,21 & 0,73 & 3,53 \\
\hline Volumen emisor (L) & 17,7 & 17,7 & 17,7 & 176,8 \\
\hline Emisores (árbol ${ }^{-1}$ ) & 10 & 10 & 10 & 1 \\
\hline Volumen ( $\mathrm{L}$ árbol ${ }^{-1}$ ) & 177 & 177 & 177 & 176,8 \\
\hline Radio mojado $(\mathrm{cm})$ & 29 & 38 & 53 & 235 \\
\hline Área mojada $\left(\mathrm{m}^{2}\right)$ & 0,26 & 0,45 & 0,88 & 17,35 \\
\hline
\end{tabular}

Determinación y manejo del VSH en las pruebas de campo

Para determinar la forma y tamaño del VSH formado por cada emisor, se utilizó el denominado 'Equipo Medidor de Volumen de Suelo Húmedo (EMVSH)' (Gispert, 2008) basado en la metodología de prospección geoeléctrica de Wenner, que evalúa la resistividad eléctrica con corrección por temperatura del suelo. La prueba de campo, previa al establecimiento de la experiencia, consistió en establecer 3 repeticiones de los 3 tipos de goteros $\left(4,8\right.$ y $\left.24 \mathrm{~L} \mathrm{~h}^{-1}\right)$ y 1 tipo de difusor $(50 \mathrm{~L}$ $\left.\mathrm{h}^{-1}\right)$, variando el tiempo de riego en función del caudal suministrado por cada uno de ellos y aplicar el mismo volumen de agua para cada tipo de emisor (177 L). Las lecturas de resistividad eléctrica se consideraron para una temperatura del suelo alrededor de $20^{\circ} \mathrm{C}$. El 
contorno del VSH se localizó cuando se observó un incremento de resistividad de 2000 $\Omega m$ que correspondían a descensos en el contenido de humedad alrededor del $3 \%$, siendo el contenido volumétrico medio del agua en el suelo del $21,5 \%$ al inicio de las pruebas.

La presencia de una capa de grava alóctona a $60 \mathrm{~cm}$ de profundidad impidió definir exactamente la profundidad máxima alcanzada por el VSH generado por cada tipo de emisor. Al efecto se consideró para todos los emisores $\left(4,8,24\right.$ y $\left.50 \mathrm{~L} \mathrm{~h}^{-1}\right)$ una profundidad de refe- rencia del frente de humedad de $60 \mathrm{~cm}$. Para los distintos tiempos de riego los valores alcanzados por un emisor en relación al radio y área mojada se indican en la Tabla 1 y los contenidos de agua a varias profundidades y distancias del emisor se presentan en la Tabla 2. Para estimar el VPER se consideró el cilindro de suelo situado debajo de la copa del árbol, que tiene por base la proyección de esta copa y por altura la profundidad estimada de las raíces. Se realizaron un total de 10 mediciones aleatorias para determinar el radio medio de proyección de la copa $(2,59 \mathrm{~m})$. La

Tabla 2. Contenido volumétrico de agua (\%) medido a distintas profundidades y distancias horizontales al eje de proyección vertical bajo el emisor, a la finalización del riego después de la aportación de los volúmenes indicados en la Tabla 1

Table 2. Volumetric water content (\%) measured at different depths and horizontal distances at projection vertical axis under the emitter, at the end of irrigation after the contribution of the volumes shown in Table 1

\begin{tabular}{lrcccc}
\hline \multirow{2}{*}{ Profundidad $(\mathrm{cm})$} & Distancia al eje vertical $(\mathrm{cm})$ & \multicolumn{5}{c}{ Caudal (L/h) } \\
\cline { 3 - 6 } & & 4 & 8 & 24 & 50 \\
\hline 10 & 0 & 30,8 & 32,0 & 34,3 & 26,4 \\
& 50 & 25,1 & 28,2 & 28,7 & \\
& 100 & 20,9 & 19,8 & 22,2 & \\
& 200 & & & & 23,6 \\
& 0 & & & & 22,1 \\
\hline 30 & 20 & 28,8 & 29,1 & 29,8 & 23,9 \\
& 50 & 23,2 & 26,9 & 29,6 & \\
& 100 & 20,0 & 20,7 & 24,4 & \\
& 200 & & & & 23,2 \\
& 0 & & & & 23,8 \\
\hline 50 & 20 & 26,1 & 25,3 & 23,1 & 23,0 \\
& 50 & 21,3 & 24,1 & 23,9 & \\
& 100 & 18,7 & 19,9 & 19,0 & \\
& 200 & & & & 21,1 \\
& & & & & 20,0 \\
\hline
\end{tabular}


profundidad radicular considerada fue de $0,60 \mathrm{~m}$ que se evaluó por medio de tres muestreos aleatorizados; bajo la copa de tres avellanos, mediante catas de $0,60 \mathrm{~m}$ de profundidad y $1 \mathrm{~m}$ de ancho. El área sombreada del avellano $\left(21 \mathrm{~m}^{2}\right)$ se obtuvo asumiendo una forma circular y considerando el radio de proyección de la copa. EI VPER $\left(12,6 \mathrm{~m}^{3}\right)$ se obtuvo como producto del área sombreada por la profundidad radicular. En la Tabla 3, para los diversos goteros y difusor utilizados, se muestran los VSH expresados en porcentaje, calculados a partir de la relación 100 $x \operatorname{VSH}\left(\mathrm{m}^{3}\right)$ NPPER $\left(\mathrm{m}^{3}\right)$.

Tabla 3. Área mojada $\left(A_{m}\right)$, área mojada respecto al área sombreada $\left(A_{s}\right)$ y volumen de suelo húmedo (VSH) expresado en unidades de capacidad y en porcentaje, para el diferente número de emisores usado en el estudio

Table 3. Wet area $\left(A_{m}\right)$, wet area regarding the shaded area $\left(A_{s}\right)$ and wet soil volume (VSH) expressed in units of capacity and percentage, for the different number of emitters used in this study

\begin{tabular}{lccccc}
\hline Caudal emisor $\left(\mathrm{L} \mathrm{h}^{-1}\right)$ & $\mathrm{N}^{\circ}$ emisores $-\mathrm{n}-$ & $\mathrm{A}_{\mathrm{m}}\left(\mathrm{m}^{2}\right)$ & $\mathrm{A}_{\mathrm{m}} / \mathrm{A}_{\mathrm{s}}(\%)$ & $\mathrm{VSH}\left(\mathrm{m}^{3}\right)$ & VSH $(\%)$ \\
\hline 4 & 10 & 2,64 & 12,53 & 0,80 & 6 \\
8 & 10 & 4,53 & 21,50 & 1,90 & 15 \\
24 & 10 & 8,82 & 41,86 & 4,10 & 33 \\
50 & 1 & 17,34 & 82,29 & 8,78 & 70 \\
\hline
\end{tabular}

\section{Producción}

En la cosecha se determinó la producción total, pesando individualmente la carga de cada uno de los 3 árboles que constituían una parcela elemental, para cada uno de los distintos porcentajes de VSH y en las 4 repeticiones, para la totalidad de la parcela experimental.

\section{Crecimiento vegetativo}

Para asegurar que el tamaño inicial de los árboles era similar en todos los tratamientos se calculó el volumen de copa al inicio del experimento. Así pues, fueron evaluados un total de 16 árboles, uno de cada parcela experimental. Se consideró la forma de la copa similar a un elipsoide con diferentes radios (altura vertical de la copa, anchura horizontal en el sentido de la fila de árboles, anchura horizontal perpendicular a la fila). Las medidas horizontales fueron realizadas usando una cinta plegable de $5 \mathrm{~m}$ (Lufkin Ultralok), y para la vertical una mira plegable de $8 \mathrm{~m}$ (Telefix). La media de los radios permitió calcular el volumen de copa.

En cada porcentaje de VSH se controló el crecimiento de 2 brotes anuales de cada uno de los 3 árboles (sobre el eje Norte-Sur) que constituían la parcela elemental de cada una de las 4 repeticiones ( 24 brotes por porcentaje de VSH). Esos brotes fueron medidos en su evolución a lo largo del período primaveral (abril, mayo, junio) durante las campañas 1994 y 1995.

\section{Parámetros de calidad del fruto}

Los parámetros de calidad fueron evaluados en muestras de frutos recogidos en el mes de 
septiembre, período de recolección. Se efectuó el control del peso de un fruto $(\mathrm{g})$ (1992 y 1994) y el rendimiento en grano (\%) (1992, 1994 y 1995).

Ambos parámetros se evaluaron a partir de una muestra de 60 avellanas recogidas de la cosecha correspondiente a 3 árboles de cada parcela elemental. De esta muestra se determinó el peso en cáscara, peso en grano, frutos vacíos y frutos dañados (ej. podredumbre).

El peso de un fruto de avellana se calculó por la relación entre el peso total del grano ( $\mathrm{g}$ ) de la muestra y el número de frutos (60 avellanas). Asimismo, el rendimiento en grano (\%) se calculó al establecer la relación entre el peso total del grano de la muestra respecto a su peso en cáscara.

\section{Productividad del agua de riego}

La productividad del agua $\left(\mathrm{P}_{\mathrm{a}}\right)$ fue definida por la relación entre la producción total de producto vendible $(\mathrm{P}, \mathrm{kg} / \mathrm{ha})$ respecto al volumen de agua aplicada mediante riego $\left(\mathrm{U}, \mathrm{m}^{3} / \mathrm{ha}\right)$,

$$
P a=\frac{P}{U}
$$

Análisis estadístico

Los resultados fueron analizados estadísticamente usando el programa SAS (SAS, Inst., 1996), estructurando los tratamientos en 4 bloques al azar y en cada uno de ellos cuatro porcentajes de VSH $(6,15,33$ y $70 \%)$ con tres repeticiones por bloque y porcentaje.

Este modelo permitió analizar el efecto de cada porcentaje de VSH sobre la producción de avellana y la comparación entre los cuatro porcentajes.

El modelo estadístico empleado fue:

donde,
$Y_{\mathrm{ijk}}$ : observación del árbol i-ésimo del año jésimo sometido al volumen de suelo húmedo k-ésimo $\left(\mathrm{VSH}_{\mathrm{k}}\right)$

$\mu$ : valor medio de la variable observada

$A_{j}$ : efecto año $(1992,1994,1995)$

$\mathrm{VSH}_{\mathrm{k}}$ : efecto del volumen de suelo húmedo $(6,15,33$ y $70 \%)$

$\mathrm{i}=1,2,3,4$

e: error

Se realizó un análisis de la varianza y un test de separación de medias (test de Duncan), para un nivel de significación $\alpha=0,05$.

\section{Resultados y discusión}

\section{Características y desarrollo de los VSH}

Las distintas descargas de los emisores $(4,8$ y $\left.24 \mathrm{~L} \mathrm{~h}^{-1}\right)$ y el difusor $\left(50 \mathrm{~L} \mathrm{~h}^{-1}\right)$ generaron diferencias en la posición del frente de humedad en los distintos VSH formados. En este sentido, se observa que una mayor descarga en el emisor generó un mayor radio mojado (Tabla 1), lo cual coincide con un comportamiento ya observado por otros autores (Bresler, 1978; Keller y Bliesner, 1990; Angelakis et al., 1993, Gispert y García, 1994; Gispert, 2008). Asimismo, el contenido volumétrico de agua en el suelo presentó, por una parte, valores crecientes a medida que aumentó la descarga del emisor y por otra parte, mostró un gradiente disminuyendo desde el interior del VSH hacia la periferia (Tabla 2), lo cual coincide con las observaciones de Gispert y García (1994) y Gispert (2008).

Evapotranspiración, pluviometría y volumen de agua de riego aplicado

La evapotranspiración media anual durante el período experimental (marzo a septiembre) se muestra en la Tabla 4. 
Tabla 4. Evapotranspiración de referencia $\left(\mathrm{ET}_{\mathrm{o}}\right)$, pluviometría efectiva $\left(\mathrm{P}_{\mathrm{e}}\right)$ y pluviometría total $\left(\mathrm{P}_{\mathrm{t}}\right)$ durante el período experimental (marzo a septiembre) en 1992-1995

Table 4. Reference evapotranspiration $\left(E T_{o}\right)$, effective precipitation $\left(P_{e}\right)$, and total precipitation $\left(P_{t}\right)$ during the experimental period (March to September) in 1992-1995

\begin{tabular}{lcccc}
\hline & 1992 & 1994 & 1995 & Media y desviación típica \\
\hline $\mathrm{ET}_{\mathrm{o}}(\mathrm{mm})$ & 913,2 & 868,9 & 831,4 & $871,2 \pm 40,9$ \\
$\mathrm{P}_{\mathrm{e}}(\mathrm{mm})$ & 370,1 & 170,9 & 159,7 & $233,6 \pm 118,4$ \\
$\mathrm{P}_{\mathrm{t}}(\mathrm{mm})$ & 462,7 & 213,7 & 199,6 & $292,0 \pm 148,0$ \\
\hline
\end{tabular}

La dosis de riego para los cuatro porcentajes de VSH fue muy similar, tal como se indica en la Tabla 5, alcanzando un valor medio para el período estudiado de $282 \mathrm{~mm}$. Esta aportación de agua de riego es muy similar a la indicada en otros trabajos en avellano (Tous et al., 1987 y Cristofori et al., 2010), en Cataluña e Italia, respectivamente.

\section{Producción de avellana}

En la Tabla 6, se observan los valores medios obtenidos del período analizado, resultando que el porcentaje más bajo de suelo húmedo
(6\%) generó de forma significativa una producción en cáscara inferior al resto de los tratamientos. No hubo diferencias significativas entre los tratamientos VSH-15\%, VSH$33 \%$ y VSH- $70 \%$.

El tratamiento $\mathrm{VSH}-70 \%$ que inicia el período experimental (1992) con una alta producción en cáscara al final del período (1995) llega a la producción menor, muy similar al tratamiento VSH-6\%.

La mayor defoliación observada durante los años 1994 y 1995, sobre el suelo debajo los avellanos que se regaron por difusión (VSH-

Tabla 5. Volumen de agua de riego aplicado (L/árbol) para 4 porcentajes distintos de volumen de suelo húmedo (VSH), durante el período 1992 a 1995

Table 5. Volume of irrigation water applied (L/tree) for 4 percentages of VSH during the period 1992 to 1995

\begin{tabular}{lcccc}
\hline VSH (\%) & 1992 & 1994 & 1995 & Media y desviación típica \\
\hline 6 & 4830 & 10505 & 8906 & $8080 \pm 2926$ \\
15 & 4820 & 10510 & 8911 & $8080 \pm 2935$ \\
33 & 4849 & 10525 & 8930 & $8101 \pm 2927$ \\
70 & 4825 & 10508 & 8909 & $8081 \pm 2931$ \\
\hline Media y desviación típica & $4831 \pm 13$ & $10512 \pm 9$ & $8914 \pm 11$ & $8085 \pm 2498$ \\
\hline
\end{tabular}

Goteo (VSH-6\%,VSH-15\%,VSH-33\%) y difusión (VSH-70\%). 
Tabla 6. Producción de avellana en cáscara $(\mathrm{kg} / \mathrm{ha})$ y productividad del agua $\left(\mathrm{P}_{\mathrm{a}}, \mathrm{kg}\right.$ avellana en cáscara/m³ agua de riego aplicada) durante los 3 años de estudio (1992, 1994 y 1995), para los distintos porcentajes de volumen de suelo húmedo (VSH)

Table 6. Production of hazelnut in shell ( $\mathrm{kg} / \mathrm{ha}$ ) and water productivity $(\mathrm{Pa}, \mathrm{kg}$ hazelnut in shell/m³ irrigation water applied) during the 3 years of study (1992, 1994 and 1995), for different percentages of wet soil volume (VSH)

\begin{tabular}{lcccc}
\hline \multicolumn{5}{c}{ Producción de avellana en cáscara (kg/ha) } \\
\hline VSH (\%) & 1992 & 1994 & 1995 & Media \\
\hline 6 & $3754,4 \mathrm{c}$ & $3429,0 \mathrm{~b}$ & $2238,2 \mathrm{c}$ & $3140,7 \mathrm{~b}$ \\
15 & $4295,9 \mathrm{bc}$ & $4259,8 \mathrm{a}$ & $3176,8 \mathrm{~b}$ & $3910,8 \mathrm{a}$ \\
33 & $3754,4 \mathrm{c}$ & $4332,0 \mathrm{a}$ & $3862,7 \mathrm{a}$ & $3983,0 \mathrm{a}$ \\
70 & $5631,6 \mathrm{a}$ & $3682,2 \mathrm{ab}$ & $2310,1 \mathrm{c}$ & $3874,6 \mathrm{a}$ \\
Media & $4359,0 \mathrm{a}$ & $3925,8 \mathrm{~b}$ & $2896,9 \mathrm{c}$ & 3727,7 \\
\hline & & $\mathrm{P}_{\mathrm{a}}\left(\mathrm{kg}\right.$ avellana en cáscara/m ${ }^{3}$ agua de riego) & \\
\hline VSH (\%) & 1992 & 1994 & 1995 & Media \\
\hline 6 & $2,15 \mathrm{~b}$ & $0,90 \mathrm{~b}$ & $0,70 \mathrm{~b}$ & $1,25 \mathrm{~b}$ \\
15 & $2,47 \mathrm{ab}$ & $1,12 \mathrm{a}$ & $0,99 \mathrm{ab}$ & $1,53 \mathrm{ab}$ \\
33 & $2,14 \mathrm{~b}$ & $1,14 \mathrm{a}$ & $1,20 \mathrm{a}$ & $1,49 \mathrm{ab}$ \\
70 & $3,23 \mathrm{a}$ & $0,97 \mathrm{ab}$ & $0,72 \mathrm{~b}$ & $1,64 \mathrm{a}$ \\
Media & $2,50 \mathrm{a}$ & $1,03 \mathrm{~b}$ & $0,90 \mathrm{~b}$ & 1,48 \\
\hline
\end{tabular}

Dentro de cada variable, valores en una misma columna con diferente letra difieren $(P<0,05)$ con relación al porcentaje de VSH. Dentro de cada variable, para las medias anuales, valores en una misma fila con diferente letra difieren $(P<0,05)$ entre años.

Goteo (VSH-6\%, VSH-15\%, VSH-33\%) y difusión (VSH-70\%).

$70 \%$ ) respecto al resto de los tratamientos, ya marcó un mayor estrés del cultivo en esas condiciones de riego, lo cual no coincide con las conclusiones de otros trabajos realizados en Italia para esta especie (De Salvador y Monastra, 1997). Por otra parte, en la prueba de campo realizada (Tabla 2) también se observa que los contenidos de agua alcanzados en el VSH del emisor por difusión son más bajos en relación con los goteros, como consecuencia de aplicar el agua en pulverización sobre una amplia superficie y posiblemente hubo una mayor pérdida de agua por evaporación di- recta desde el suelo. La baja resistencia al estrés que posee el avellano (Natali et al., 1988; Bignami y Natali, 1992; Mingeau et al., 1994; Tombesi, 1994) que reduce sensiblemente su capacidad productiva (Girona et al., 1994), puede ser la causa más probable de la menor producción alcanzada por el porcentaje VSH$70 \%$, respecto a VSH-15\% y VSH-33\%, durante la mayoría de años (1994 y 1995).

Sin embargo, el tratamiento más insatisfactorio, desde el punto de vista productivo, fue VSH-6\%, significativamente inferior al resto por el menor VSH generado. También el go- 
tero de $4 \mathrm{~L} \mathrm{~h}^{-1}$, que corresponde a ese porcentaje, fue el que presentó un menor contenido volumétrico medio de agua, respecto a los otros goteros $\left(8\right.$ y $24 \mathrm{~L} \mathrm{~h}^{-1}$ ) al finalizar la prueba de campo (Tabla 2). Debe descartarse la influencia en los resultados de pérdidas por percolación profunda diferenciadas en el tratamiento VSH-6\% que por emitir un caudal menor requirió mayor tiempo de riego. En efecto, atendiendo a los valores de contenido de agua mostrados en la Tabla 2 para el caso de $\mathrm{VSH}-6 \%$ (emisor $4 \mathrm{~L} \mathrm{~h}^{-1}$ ), al final del riego, solo son un $3 \%$ superiores a VSH-33\% (emisor $24 \mathrm{~L} \mathrm{~h}^{-1}$ ) para la profundidad de $50 \mathrm{~cm}$ por debajo del emisor, mientras que a $10 \mathrm{~cm}$ de profundidad VSH-6\% son un 3,5\% inferiores a VSH-33\%, por lo que la tendencia durante el periodo de redistribución iría en el sentido de compensarse los contenidos de agua.

Al comparar las producciones en cáscara obtenidas en las diferentes campañas (1992, 1994 y 1995), se observa que la producción estuvo muy influenciada por la pluviometría anual. Así pues en 1992, año con elevada pluviometría total entre marzo y septiembre se alcanzó la mayor producción en cáscara, mientras que en 1995 con escasa pluviometría (Ta- bla 4), se obtuvo la menor producción (Tabla 6). Al ser el avellano un cultivo más adecuado para un hábitat más húmedo (Farquhar et al., 1980), con elevada humedad relativa y primaveras lluviosas, tuvo una respuesta más favorable en el año 1992 por la lluvia primaveral (283,6 mm) que en 1995 en que la primavera fue más seca $(93,4 \mathrm{~mm})$. A pesar del agua aportada por el riego, en este último año el avellano sufrió más debido a los elevados déficits de presión de vapor de la atmósfera. El avellano, en estas circunstancias meteorológicas, manifiesta síntomas de estrés aun aplicando los riegos requeridos según su ETc.

Asimismo, hay que hacer mención a las diferencias del riego estacional entre años con cantidades que oscilan entre $4831 \mathrm{~L}$ árbol (1992) y 10512 L/árbol (1994), como consecuencia de la variabilidad pluviométrica de estos años (Tabla 4).

\section{Crecimiento vegetativo}

Los valores que se presentan en la Tabla 7 permiten apreciar la existencia de diferencias significativas entre porcentajes de VSH, para 1994

Tabla 7. Longitud total (mm) del brote anual del avellano durante los años 1994-1995, para los distintos porcentajes de volumen de suelo húmedo (VSH)

Table 7. Total length ( $\mathrm{mm}$ ) of hazelnut annual shoot during 1994-1995 for different percentages of wet soil volume (VSH)

\begin{tabular}{cccc}
\hline & \multicolumn{3}{c}{ Longitud total del brote anual $(\mathrm{mm})$} \\
\hline VSH (\%) & 1994 & 1995 & Media \\
\hline 6 & $105,2 \mathrm{bc}$ & 67,3 & $86,3 \mathrm{bc}$ \\
15 & $127,3 \mathrm{ab}$ & 67,2 & $97,3 \mathrm{~b}$ \\
33 & $154,3 \mathrm{a}$ & 84,8 & $119,5 \mathrm{a}$ \\
70 & $92,0 \mathrm{c}$ & 60,6 & $76,3 \mathrm{c}$ \\
Media & $119,7 \mathrm{a}$ & $70,0 \mathrm{~b}$ & 94,8 \\
\hline
\end{tabular}

Valores en una misma columna con diferente letra difieren $(P<0,05)$ con relación al porcentaje de VSH. Valores en la fila de las medias con letra diferente difieren $(P<0,05)$ entre años. Goteo (VSH-6\%, VSH-15\%, VSH-33\%) y difusión (VSH-70\%). 
y en el promedio de todo el período analizado (1994 y 1995). El porcentaje VSH-33\% destacó significativamente respecto a los otros tratamientos. Asimismo VSH-70\% es el que genera menor crecimiento vegetativo al igual que ocurrió con la producción de avellana en cáscara.

Las diferencias vegetativas observadas entre años (1994 y 1995), mantienen un paralelismo respecto a la aportación pluviométrica anual. En 1995, año de escasa lluvia (Tabla 4) se produjo el menor crecimiento vegetativo $(70,0$ $\mathrm{mm}$ ), siendo en 1994, con mayor pluviometría (Tabla 4), cuando el brote anual tuvo un crecimiento significativo superior $(119,7 \mathrm{~mm})$.

El fruto es el sumidero principal de carbohidratos (Bloom et al., 1985), lo cual va en detrimento del desarrollo fisiológico de otros órganos de la planta como brotes anuales, desarrollo del tronco y en último lugar la raíz (Grossman y Dejong, 1994). Por todo ello, otra posible causa que originó un menor crecimiento vegetativo durante 1995 puede ser la alternancia productiva, después del año 1994 , con buena cosecha (3926 kg/ha), que redujo la acumulación de reservas generando un descenso de cosecha y de crecimiento vegetativo el año siguiente.

\section{Parámetros de calidad}

\section{Peso de 1 grano de avellana}

Según se observa en la Tabla 8 el grano de avellana alcanzó su mayor peso al establecer un porcentaje de suelo húmedo del $33 \%$, especialmente durante 1992 en que la diferencia fue significativa respecto a los otros porcentajes de VSH. Los VSH del $6 \%$ y el $70 \%$ de VSH obtienen una respuesta muy similar respecto al peso del grano. Sin embargo, los motivos que explicarían tal comportamiento son diferentes.

Tabla 8. Peso (g) de 1 grano de avellana durante los años 1992 y 1994, para los diferentes porcentajes de volumen de suelo húmedo (VSH) Table 8. Average kernel weight (g) during the years 1992 and 1994, for different percentages of wet soil volume (VSH)

\begin{tabular}{cccl}
\hline & \multicolumn{3}{c}{ Peso de 1 grano de avellana $(\mathrm{g})$} \\
\hline VSH (\%) & 1992 & 1994 & Media \\
\hline 6 & $0,70 \mathrm{~b}$ & $0,52 \mathrm{~b}$ & $0,61 \mathrm{c}$ \\
15 & $0,75 \mathrm{~b}$ & $0,65 \mathrm{ab}$ & $0,70 \mathrm{~b}$ \\
33 & $0,84 \mathrm{a}$ & $0,73 \mathrm{a}$ & $0,78 \mathrm{a}$ \\
70 & $0,62 \mathrm{c}$ & $0,53 \mathrm{~b}$ & $0,58 \mathrm{c}$ \\
Media & $0,73 \mathrm{a}$ & $0,61 \mathrm{~b}$ & 0,67 \\
\hline
\end{tabular}

Valores en una misma columna con diferente letra difieren $(P<0,05)$ con relación al porcentaje de VSH. Valores en la fila de las medias con letra diferente difieren $(\mathrm{P}<0,05)$ entre años. Goteo (VSH-6\%, VSH-15\%, VSH-33\%) y difusión (VSH-70\%). 
En el caso de VSH-6\% el comportamiento productivo fue consecuencia del escaso volumen de suelo mojado proporcionado, que se manifestó con mayor intensidad en 1994 por ser un año más seco. En cambio, el mal comportamiento de VSH- $70 \%$ no es atribuible al escaso VSH generado sino al sistema de aportación del riego por difusión y al bajo contenido volumétrico de agua en el suelo que ese sistema ocasionó, generando defoliación prematura del cultivo y estrés altamente perjudicial.

Al comparar entre años se observa que en 1992 tanto la precipitación ocurrida entre marzo y septiembre como la precipitación en primavera fueron muy superiores a la de 1994, permitiendo alcanzar un mayor peso del grano. La influencia del agua primaveral como factor favorable para la calidad del grano ya fue observada por Gispert et al. (2005) en un estudio experimental sobre avellano.

\section{Rendimiento en grano}

La Tabla 9, muestra la existencia de diferencias significativas entre porcentajes de VSH.
Al considerar en su totalidad los valores medios del período experimental (1992-1995) se observa que los tratamientos VSH-33\% y VSH-15\% son superiores en rendimiento en grano respecto al tratamiento VSH-6\%. Estos datos de rendimiento de avellana de la variedad 'Pauetet', son similares a los indicados por otros autores (IRTA, 1997; Germain y Sarraquigne, 2004), con un valor del $48 \%$.

Al igual que sucedió para la media de los años 1992 y 1994 en que el mayor peso de 1 grano de avellana se producía para el tratamiento VSH-33\%, para el rendimiento en grano también se alcanza el máximo valor para el mismo porcentaje de suelo húmedo (33\%) que con el tratamiento VSH-15\% superan de forma significativa el rendimiento obtenido con el porcentaje más reducido (VSH-6\%).

Las condiciones meteorológicas del año influyen en el rendimiento productivo del grano al variar el contenido de frutos vacíos o podridos. Durante los años húmedos, si no se hace un buen secado del fruto en cáscara, suelen incrementarse los problemas de granos podridos por mohos. Asimismo, durante los

Tabla 9. Rendimiento en grano (\%) de los frutos de avellana durante los años 1992, 1994 y 1995, para los diferentes porcentajes de volumen de suelo húmedo (VSH) Table 9. Grain yield (\%) of fruit hazelnut during the years 1992, 1994 and 1995, for different percentages of wet soil volume (VSH)

\begin{tabular}{ccccl}
\hline & \multicolumn{4}{c}{ Rendimiento en grano (\%) } \\
\hline VSH (\%) & 1992 & 1994 & 1995 & Media \\
\hline 6 & $46,79 \mathrm{ab}$ & $44,82 \mathrm{c}$ & $50,55 \mathrm{~b}$ & $47,56 \mathrm{~b}$ \\
15 & $48,92 \mathrm{a}$ & $48,15 \mathrm{ab}$ & $49,48 \mathrm{c}$ & $48,83 \mathrm{a}$ \\
33 & $47,75 \mathrm{a}$ & $49,94 \mathrm{a}$ & $48,88 \mathrm{c}$ & $49,17 \mathrm{a}$ \\
70 & $42,96 \mathrm{~b}$ & $46,64 \mathrm{bc}$ & $51,53 \mathrm{a}$ & $48,21 \mathrm{ab}$ \\
Media & $46,60 \mathrm{~b}$ & $47,39 \mathrm{~b}$ & $50,11 \mathrm{a}$ & 48,44 \\
\hline
\end{tabular}

Valores en una misma columna con diferente letra difieren $(P<0,05)$ con relación al porcentaje de VSH. Valores en la fila de las medias con letra diferente difieren $(P<0,05)$ entre años. Goteo (VSH-6\%, VSH-15\%, VSH-33\%) y difusión (VSH-70\%). 
años secos, las mermas suelen ser ocasionadas por una mayor presencia de avellanas vacías que se desprenden fácilmente del involucro antes de alcanzar su madurez y, también, tienen un menor desarrollo en cáscara. Así pues, durante la campaña de 1992, que fue más lluviosa se observó un menor porcentaje de frutos vacíos $(3 \%)$ en las muestras analizadas, mientras que durante 1995, año más seco, ese porcentaje aumentó sensiblemente (7\%).

La evolución del peso en grano por árbol para los distintos porcentajes de VSH se indica en la Figura 1 para los distintos años, evidenciándose una progresiva adaptación de la respuesta productiva del cultivo al incremento del porcentaje de VSH a medida que avanza el período experimental. Este mismo comportamiento fue observado por Gispert et al. (2013) en olivos regados por goteo. En el año 1992 existe un cierto desorden productivo que se va modificando durante los siguientes años, sobretodo al final del período experimental en que el porcentaje comprendido entre 15 y $33 \%$ de VSH generan la mayor respuesta productiva.

\section{Productividad del agua de riego}

La Tabla 6 muestra importantes diferencias entre los valores de productividad del agua de riego por años, siendo ésta máxima en el año 1992 y significativamente distinta a la del resto de años, puesto que 1992 fue el año de mayor producción de avellana, mayor preci-

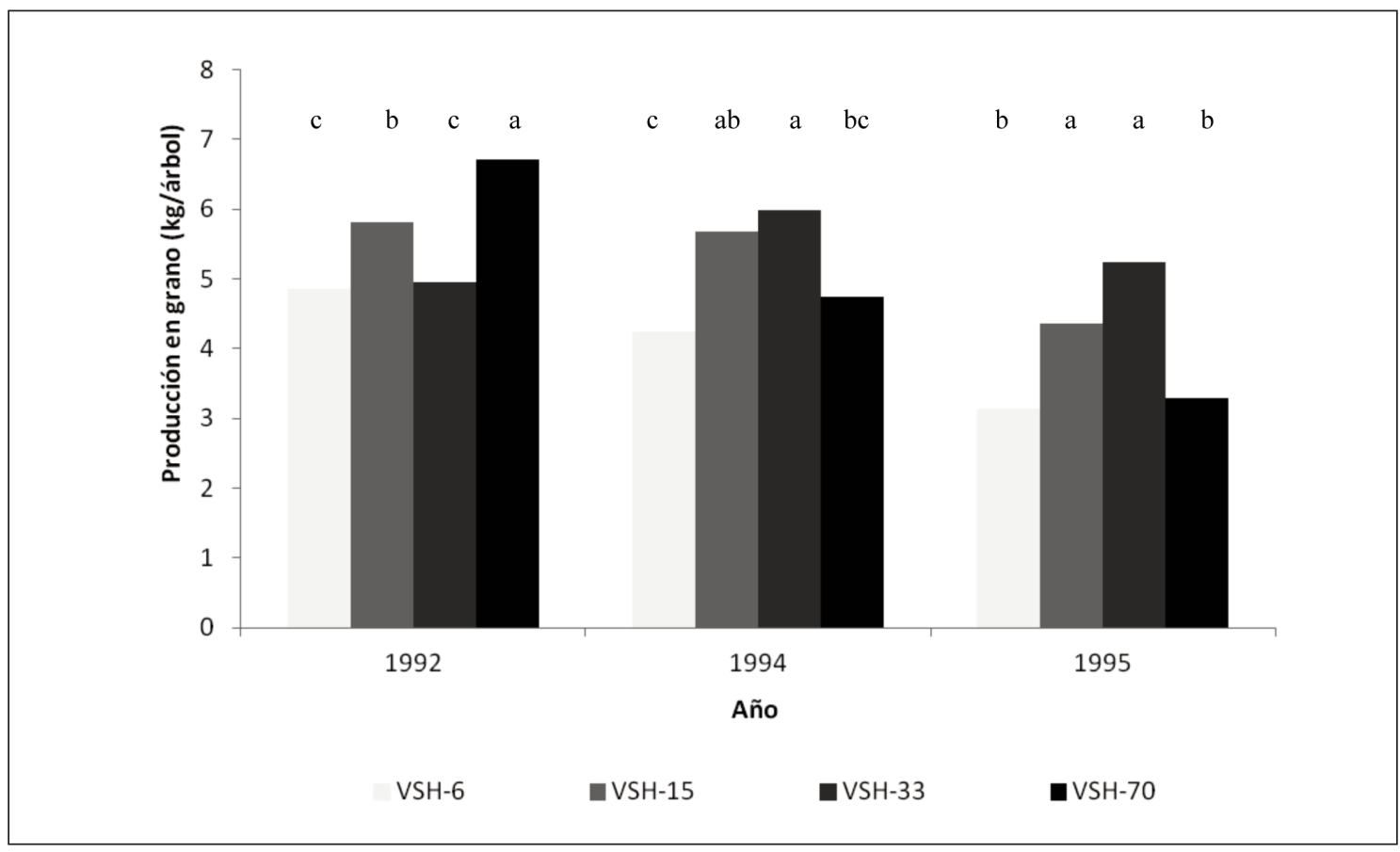

Figura 1. Efecto del porcentaje de VSH sobre la producción en grano (kg/árbol) a lo largo del período experimental (1992, 1994 y 1995). Goteo (VSH-6\%, VSH-15\%, VSH-33\%) y difusión (VSH-70\%). Figure 1. Effect of the VSH percentage on the production of grain ( $\mathrm{kg} /$ tree) throughout the experimental period (1992, 1994 and 1995). Drip (VSH-6\%, VSH-15\%, VSH-33\%) and micro-sprayer (VSH-70\%). 
pitación y menor volumen de agua de riego aplicado. Considerando el conjunto de los años de ensayo, aunque el VSH-33 presentó el menor valor de productividad del agua de riego éste no fue significativamente distinto del correspondiente a los restantes volúmenes de suelo húmedo obtenidos con goteo. El valor medio considerando conjuntamente los 4 porcentajes de VSH, en el período experimental (1992-1995), fue similar al obtenido por Tombesi y Rosati (1997) en trabajos realizados en Italia.

\section{Conclusiones}

El menor porcentaje de volumen de suelo húmedo ensayado (VSH-6\%), normalmente utilizado en las explotaciones comerciales de avellano de Tarragona, generó de forma significativa una producción en cáscara inferior al resto de los tratamientos estudiados (VSH$15 \%$, VSH-33\% y VSH-70\%), a pesar de recibir la misma cantidad de agua de riego. Las posibles causas de este comportamiento hay que atribuirlas al menor contenido volumétrico medio de agua en el suelo y al menor volumen de suelo húmedo a disposición del sistema radicular.

La aplicación del agua de riego por difusión generó el mayor porcentaje de suelo húmedo (VSH-70\%), pero con menor contenido de agua en el suelo, probablemente por una mayor evaporación desde su superficie, ocasionando manifestaciones de estrés hídrico como defoliación prematura y producciones en cáscara similares a las obtenidas con el porcentaje más bajo de suelo húmedo (VSH-6\%).

El mayor crecimiento vegetativo se alcanzó con el porcentaje del $33 \%$, sin embargo no se correspondió con una diferencia significativa en mayor producción en cáscara $(\mathrm{kg} / \mathrm{ha}$ ) respecto de los tratamientos de $\mathrm{VSH}-15 \%$, VSH-33\% y VSH-70\%.
A lo largo del experimento se observó una progresiva adaptación de la respuesta productiva del cultivo al incremento del porcentaje de VSH.

En relación con los parámetros de calidad, la mejor respuesta del cultivo en relación al peso de 1 grano de avellana (g) correspondió al tratamiento $\mathrm{VSH}-33 \%$, mientras que los porcentajes VSH-6\% y VSH-70\% generaron pesos menores.

El mayor rendimiento en grano (\%) se obtuvo para porcentajes de suelo húmedo del 15 al $33 \%$ los cuales fueron significativamente superiores al alcanzado por VSH-6\%.

Se recomienda, para las condiciones en que se realizó el ensayo y atendiendo a los parámetros productivos y de calidad del fruto obtenidos, la utilización del riego por goteo frente al riego por difusión y la utilización de porcentajes de VSH mínimos del 33\%, que además no difieren significativamente en la eficiencia en el uso del agua de riego respecto de los tratamientos con VSH inferior.

\section{Agradecimientos}

El trabajo fue posible gracias a la financiación del Departamento de Agricultura, Ganadería y Pesca de la Generalitat de Catalunya y la colaboración del personal técnico, Sr. Jesús Gil y Sr. Àngel Companys, de la Oficina Comarcal de l'Alt Camp (Tarragona) para los trabajos de campo.

\section{Bibliografía}

Arbat G, Barragán J, Puig-Bargués J, Poch R, Ramírez de Cartagena F (2003). Evaluación de los modelos numéricos de flujo de agua en el suelo HYDRUS-2D y SIMDAS en riego localizado. Estudios de la Zona No Saturada del Suelo. Vol. VI, pp. 279-288. 
Arbat G, Puig-Bargués J, Duran-Ros M, Barragán J, Ramírez de Cartagena F (2013). Drip-Irriwater: Computer software to simulate soil wetting patterns under surface drip irrigation. Computers and Electronics in Agriculture, 98: 183-192

Allen RG, Pereira LS, Raes D, Smith M (1998). Crop evapotranspiration: Guidelines for computing crop water requirements. Irrigation and drainage 56. UN FAO, Rome, Italy, 299 p.

Angelakis A, Rolston D, Kadir T, Scott V (1993). Soil water distribution under trickle source. Journal of Irrigation and Drainage Engineering 119 (3): 484-500.

Baiges F, Ferré IR, Puig V, Secanell P, Gonzalez C, Gil J (2012). Caracterización de las explotaciones con cultivo de avellano en Cataluña. Fruticultura 21: 4-11.

Bennett Ch (2002). Developing a good almond root system with drip irrigation. Australian Nutgrower Sept-Nov: 12-18.

Bignami C, Natali S (1992). Relazioni idriche e fotosintesi di alcune specie da frutto. Atti delle Giornate Scientifiche della Società di Ortoflorofrutticoltura Italiana, Ravello (Italia), 8-10 Abril 1992: 174-175.

Bignami C, Cristofori V, Ghini P, Rugini E (2009). Effects of irrigation on growth and yield components of hazelnut (Corylus avellana L.) in Central Italy. Acta Horticulturae 845: 309-314.

Bignami C, Cristofori V, Bertazza G (2011). Effects of water availability on hazelnut yield and seed composition during fruit growth. Acta Horticulturae 922: 333-340.

Bloom A J, Chapin F S, Mooney HA (1985). Resource limitation in plants- an economic analogy. Annual Review of Ecological Systems 16: 363-392.

Bresler E, Heller J, Diner N, Ben-Asher A, Brand A, Goldberg D (1971). Infiltration from a trickle source. II. Experimental data and theoretical predictions. Soil Science Society of America Proceedings, 35: 683-689.

Bresler E (1978). Analysis of trickle irrigation with application to design problems. Irrigation Science 1, pp. 3-7.
Cohen M, Girona J, Poblet P, Fortuny J, Gené J (1992). El riego del avellano en 3 municipios representativos de Tarragona: Riudoms (Baix Camp), Alcover (Alt Camp) y Vila-Seca (Tarragonés). ITEA Vol. 88V, n² 1, pp. 31-45.

Cristofori V, Bignami C, Gasbarra S, Rugini E (2010). Irrigazione del nocciolo nel viterbese: sistema irrigui localizzati per una optimizzazione d'uso dell'acqua. ISSN 2038-8292. Corylus \&Co, Anno I, numero 2: 31-38.

De Salvador FR, Monastra F (1997). Water regime and soil management in hazelnut trees: preliminary studies in pots. Acta Horticulturae 445: 255-262.

Doorenbos J, Pruitt WO (1988). Las necesidades de agua de los cultivos. Estudio FAO Riego y Drenaje 24. $194 \mathrm{p}$.

FAOSTAT (2014a). Production, Crops. Disponible en: http://faostat.fao.org/site/567/default.aspx\#ancor, February, 2014.

FAOSTAT (2014b). Production, Value of agricultural production. Disponible en: http://faostat.fao. org/site/613/default.aspx\#ancor, February, 2014.

Farquhar GH, Schulze ED, Küppers M (1980). Responses to humidity by stomata of Nicotiana glauca and Corylus avellana L. are consistent with the optimization of carbon dioxide uptake with respect to water loss. Australian Journal of Plant Physiology. 7: 315-327.

Fereres E (1981). Papel de la fisiología vegetal en la microirrigación. Recomendaciones para el manejo mejorado. Ponencia en IV Seminario Latinoamericano de microirrigación. Barquisimeto, Venezuela. 21- 27 Junio 1981. 23 p.

Fideghelli C, De Salvador FR (2009). World hazelnut situation and perspectives. Acta Horticulturae 845: 39-52.

Fernández JE, Moreno F, Cabrera F, Arrúe JL, Martín-Aranda J (1991). Drip irrigation, soil characteristics and the root distribution and root activity of olive trees. Plant and Soil 133: 239-251.

Germain E, Sarraquigne JP (2004). Le noisetier. Ctifl. París, Francia. 296 p. 
Girona J, Cohen M, Mata M, Marsal J, Miravete C (1994). Physiological, growth and yield responses of hazelnut (Corylus avellana L.) to different irrigation regimes. Acta Horticulturae 351: 463-472.

Gispert JR, Ramírez de Cartagena F, Villar JM, Girona J (2013). Wet soil volume and strategy effects on drip-irrigated olive trees (cv. 'Arbequina'). Irrigation Science 31: 479-489.

Gispert JR (2008). Investigación para caracterizar el volumen de suelo húmedo (VSH) en riego localizado. Influencia del VSH en olivo (Olea europaea L.), manzano (Malus domestica BORK) y avellano (Corylus avellana L.). Tesis Doctoral. Universitat de Lleida, España. pp. 205-233.

Gispert JR, Tous J, Romero A, Plana J, Gil J, Company $A$ (2005). The influence of different irrigation strategies and the percentage of wet soil volume on the productive and vegetative behaviour of the hazelnut tree (Corylus aveIlana L.). Acta Horticulturae 686: 333-341.

Gispert JR, Gil J, Company A (1996).La superficie mullada del sòl en el cultiu de l'avellaner. Catalunya Rural i Agrària 29: 29-37.

Gispert JR, García J (1994). El volumen húmedo del suelo. Aspectos agronómicos relacionados con la microirrigación (II). Riegos y drenajes, XXI/77: 16-28.

Grau P (2009). Manual de Avellano Europeo. Boletín INIA, 195. Chillan, Chile. 96 p.

Grossman YL, Dejong TM (1994). Peach: A simulation model of reproductive and vegetative growth in peach trees. Tree Physiology 14: 329-345.

IRTA (1997). Fichas Varietales 'Pauetet'. El Boletín de Crisol y Ceres de Frutos Secos 5: 23.

Jiménez A, Caballero F, Funes E (1977). Aplicación de isótopos a los problemas de la fertilización. Seminario Fertilización cultivos. CRIDA-06. Madrid, España.

Keller J, Bliesner RD (1990). Sprinkle and trickle irrigation. New Cork: Van Nostrand Reinhold, 662 p.

Marsal J, Girona J, Mata M (1997). Leaf Water Relation Parameters in Almond Compared to Hazelnut Trees during a Deficit Irrigation Period. Journal of the American Society for Horticultural Science. 122(4): 582-587.
Mingeau M, Ameglio T, Pons B, Rousseau P (1994). Effects of water stress on development growth and yield of hazelnut trees. Acta Horticulturae 351: 305-314.

Ministerio de Agricultura, Alimentación y Medio Ambiente (MAGRAMA) (2013). Encuesta de superficies y rendimientos de cultivos (ESYRCE). Secretaria General Técnica. Madrid.

Natali S, Bignami C, Gonzales MJ (1988). Effetti dello stres idrico sul potenziale idrico fogliare, sulla traspirazione e sulla fotosintesi in Corylus avellana L. Irrigazione e drenaggio 3: 118-123.

Nuñez-Aguilar I, Arrúe-Ugarte JL, Moreno F, Martín-Aranda J (1980). Sustracción de humedad en la zona radicular del olivo (variedad manzanillo). Técnicas de seguimiento y primeros resultados obtenidos. Actas del VII Simposio de Bioclimatología del Centro Superior de Investigaciones Científicas. Avances sobre la investigación en Bioclimatología, Sevilla (España) Diciembre 1990: 515-524.

Romisondo P, Me G, Radicati L (1988). El cultivo moderno y rentable del avellano. Ed. De Vecchi. Barcelona, España. 126 p.

Rovira M, Aletà N (2012).Corylus avellana L. En: Producción y manejo de semillas y plantas forestales. Tomo I. (Ed. Pemán J, Navarro R, Nicolás JL, Prada MA, Serrada R.) MARM: 394-405.

SAS Institute (1996). SAS Software. SAS Inst., Cary, NC.

Schwartzmass M, Zur B (1986). Emitter spacing and geometry of wetted soil volume. Journal of Irrigation and Drainage Engineering 112 (3), 242-253.

Silva AP, Santos F, Santos A, Sousa V, Lopes A, Assunçao A, Mota B, Carvalho JL, Borges O (2005). A Aveleira. Projecto Agro 162. Vila Real, Portugal. $178 \mathrm{p}$.

Soil Survey Staff (2010). Keys to Soil Taxonomy, $11^{\text {th }}$ ed. USDA-Natural Resources Conservation Service, Washington, DC (EE. UU.).

Tombesi A, Rosati A (1997). Hazelnut response to water levels in relation to productive cycle. Fourth International Symposium on Hazelnut. Acta Horticulturae 445, 269-278. 
Tombesi A (1994). Influence of soil water levels on assimilation and water use efficiency in hazelnut. Acta Horticulturae 351: 247-255.

Tous J, Rovira M, Plana J (1987). Cultivo del aveIlano. Fruticultura Profesional 11: 115-123.

Tous J, Rovira, M, Romero A (2001). Avellano. En: La Horticultura Española (SECH). Mundi Prensa: 275-278.
Tous J, Rovira M (2004). Situación y perspectivas agronómicas del cultivo del avellano. Vida Rural 201:41-45.

(Aceptado para publicación el 19 de agosto de 2014) 